\title{
A 4 x 8-Gbps VCSEL Array Driver ASIC and Integration with a Custom Array Transmitter Module for the LHC Front-end Transmission
}

\author{
Di Guo a,b," ${ }^{\text {, }}$ Chonghan Liu ${ }^{\mathrm{a}}$, Jinghong Chen ${ }^{\mathrm{c}}$, John Chramowicz ${ }^{\mathrm{d}}$, Datao Gong ${ }^{\mathrm{a}}$, \\ Huiqin $\mathrm{He}^{\mathrm{a}, \mathrm{e}}$, Suen Hou ${ }^{\mathrm{f}}$, Tiankuan Liu ${ }^{\mathrm{a}}$, Alan Prosser ${ }^{\mathrm{d}}$, Ping-Kun Teng ${ }^{\mathrm{f}}$, \\ Annie C. Xiang ${ }^{\mathrm{a}}$, Le Xiao ${ }^{\mathrm{a}, \mathrm{g}}$ and Jingbo $\mathrm{Ye}^{\mathrm{a}}$ \\ ${ }^{a}$ Department of Physics, Southern Methodist University, Dallas, TX 75275, USA \\ ${ }^{b}$ State Key Laboratory of Particle Detection and Electronics, University of Science and Technology of China, Hefei Anhui 230026, China \\ ${ }^{c}$ Department of Electrical and Computer Engineering, University of Houston, Houston, TX 77004, USA \\ ${ }^{d}$ Real-Time Systems Engineering Department,Fermi National Laboratory, Batavia, IL 60510, USA \\ e Shenzhen Polytechnic, Shenzhen 518055, P.R. China \\ ${ }^{f}$ Institute of Physics, Academia Sinica, Nangang 11529, Taipei, Taiwan \\ ${ }^{g}$ Department of Physics, Central China Normal University, Wuhan, Hubei 430079, P.R. China
}

\begin{abstract}
This paper describes the design, fabrication and experiment results of a 4 x 8-Gbps Vertical-Cavity Surface-Emitting Laser (VCSEL) array driver ASIC with the adjustable active-shunt peaking technique and the novel balanced output structure under the Silicon-on-Sapphire (SOS) process, and a custom array optical transmitter module, featuring a compact size of $10 \mathrm{~mm} \mathrm{x}$ $15 \mathrm{~mm} \times 5.3 \mathrm{~mm}$. Both the array driver ASIC and the module have been fully tested after integration as a complete parallel transmitter. Optical eye diagram of each channel passes the eye mask at $8 \mathrm{Gbps} / \mathrm{ch}$ with adjacent channel working simultaneously with a power consumption of $150 \mathrm{~mW} / \mathrm{ch}$. The optical transmission of Bit-Error Rate (BER) less than 10E-12 is achieved at an aggregated data rate of $4 \times 8$-Gbps.
\end{abstract}

Keywords: Optical detector readout concepts; Front-end electronics for detector readout; Lasers Driver ASIC; Array optical transmission

(C) 2016. This manuscript version is made available under the Elsevier user license http://www.elsevier.com/open-access/userlicense/1.0/ 


\section{1. Introduction}

2 VCSEL-based parallel optical data links have 3 recently seen rapid and wide-spread deployment in 4 high performance computing labs, data centers and ${ }_{5}$ Ethernet networks. The benefits include highly 6 aggregated bandwidth, compact footprint and low 7 power consumption. To apply the parallel optical 8 links to the LHC upgrades as well as in other collider 9 detector developments [1], these devices have to 10 meet rigid environmental demands of radiation, 11 electro-magnetic field, temperature and volume size. 12 The high luminosity running of the LHC (HL-LHC), 13 with instantaneous luminosities of $5 \times 10^{34} \mathrm{~cm}^{-2} \mathrm{~s}^{-1}$ 14 and an integrated luminosity of $3000 \mathrm{fb}^{-1}$, for 15 example, will request that the opto-electronics 16 devices are close to the beam pipe to withstand 17 integrated radiation dose of $100 \mathrm{KGy}$ and $10^{15}$ 18 neutrons $/ \mathrm{cm}^{2}[2,3]$.

Two critical developments, the array driver ASIC 20 and the array optical coupling, are indispensable 21 towards a dedicated array optical transmitter for on22 detector deployment. Prior developments of single 23 channel optical transceivers, such as CERN's 24 versatile link project [4], have successfully qualified 25 commercial components, custom developed OE 26 circuitry and assembly packages. In advancing to 27 array-based parallel modules, limited by the channel28 to-channel pitch of extremely tight industrial 29 standard, both the array ASICs and the array optical 30 module face new customization challenges.

A family of data link ASICs has been developed 32 by SMU using a commercial $0.25-\mu \mathrm{m}$ Silicon-on33 Sapphire (SOS) CMOS technology [5]. The LOCld4 34 ASIC is a four channel, 8-Gbps/ch laser array driver 35 designed for the array optical transmitter module. ${ }_{36}$ Each channel receives the low-swing Current-Mode 37 Logic (CML) signal (200 mV differential peak-to38 peak), outputs a modulation-current signal of $7.5 \mathrm{~mA}$ 39 with an average current of $6.25 \mathrm{~mA}$ with a power 40 consumption of $150 \mathrm{~mW} / \mathrm{ch}$ at $8 \mathrm{Gbps} / \mathrm{ch}$. This is the 41 first VSCEL array driver ever fabricated at this 42 aggregated bandwidth targeting high-energy physics 43 experiments. Programmable active-shunt peaking 44 technique is used to extend the bandwidth in pre45 driving stages [6]. A novel structure of the output
46 stage removes the ordinary extra bias-circuit, and 47 delivers both bias and modulation current with 48 balanced branches to effectively minimize the noise 49 and crosstalk from the power supply.

50 After integrated within a custom array optical 51 transmitter (ATx), the LOCld4 is able to be fully 52 tested. The ATx module, while utilizing generic 53 commercial array-optical-coupling components [7], is 54 assembled via a custom low-cost reliable active55 alignment procedure. Radiation tolerance has been 56 tested at individual component level. The commercial ${ }_{57}$ VCSEL arrays (12-channel GaAs quantum well 58 surface emitter) are being irradiated continuously 59 with quantitative Non-Ionizing Energy Loss (NIEL) 60 up to $10^{15} \mathrm{~cm}^{-2} 1-\mathrm{MeV}$ neutrons equivalents by ${ }_{61}$ CERN [8]. The commercial array optical elements 62 have been exposed to X-ray up to $96 \mathrm{Mrad}\left(\mathrm{SiO}_{2}\right)$ at a 63 dose rate of $6668 \mathrm{Krad}\left(\mathrm{SiO}_{2}\right) / \mathrm{hr}$, no radiation64 induced attenuation is observed [9]. The radiation 65 tolerant performance of the SOS process has been 66 evaluated [10], and verified on a single channel ${ }_{67}$ VCSEL driver with x-ray and high-energy neutron 68 beam test [11].

69 The structure and design of the four-channel 70 VCSEL Array driver LOCld4 are discussed in 71 Section 2. The array transmitter module development 72 and optical alignment procedures are described in 73 Section 3. In Section 4, we discuss the full-channel 74 optical test of the LOCld4 after integration with the 75 ATx module. And the conclusions are presented in 76 Section 5.

\section{2. Four-channel VCSEL Array driver: LOCld4}

78 The block diagram of LOCld4 is shown in Fig. 1. 79 The common voltage (vcom) of $1.9 \mathrm{~V}$ is generated 80 and provided internally with impedance match as the 81 input interface, followed by the pre-drivers with the 82 external $3.3 \mathrm{~V}$ (Vdd) power supply and the last 83 output driver with the external $3.8 \mathrm{~V}$ (Vvcsel) power 84 supply. The pre-drivers are five-stage differential 85 amplifiers with the adjustable active-shunt peaking 86 technique for the consideration of amplification and 87 bandwidth. The peaking strength and the tail current 88 of pre-drivers, which mainly control the modulation 89 amplitude, are both adjustable through the 90 Peaking_Adj and the Iref_pre respectively, as shown 
93 Fig. 1. Block diagram of the 4-channel array driver LOCld4

94
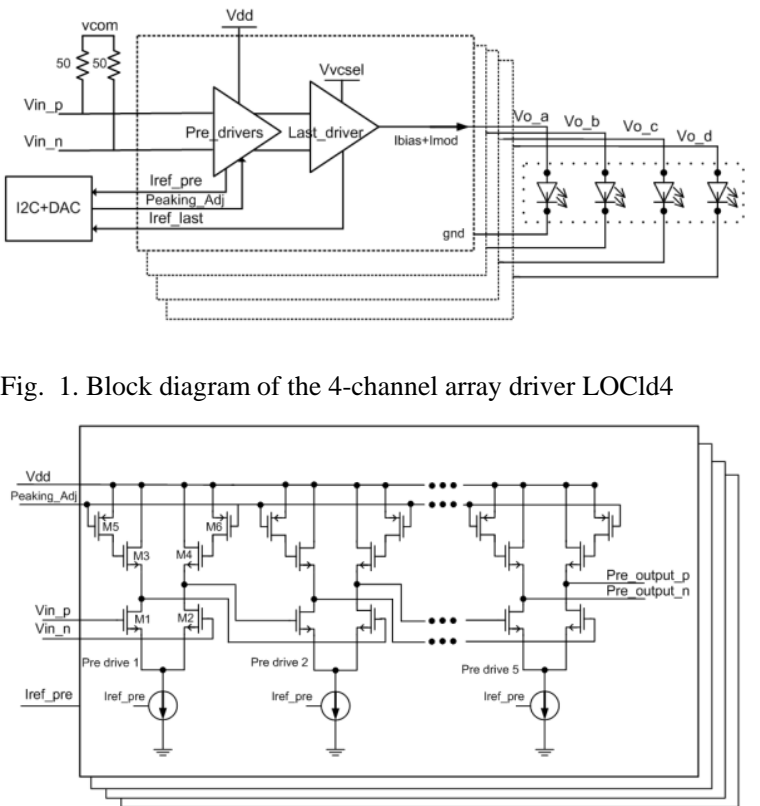

96 Fig. 2. Schematic of the pre-drivers in the LOCld4

98 in the Fig. 1.

The last driver, receiving the differential outputs 100 of pre-drivers, adopts the single-end, open-drain 101 structure to enable the DC coupling between the 102 VCSEL and the driver for the array application. It 103 combines the supply of bias and modulation current 104 without the conventional extra bias-current circuit, 105 and deliberately designs the load of internal branch to 106 match with the external branch (VCSEL load). This 107 novel "balanced" structure effectively weakens 108 switching components on the power supply of the last 109 driver, which minimizes the ground bounce noise and 110 crosstalk accordingly when compared to the 111 conventional single-end output stage.

\section{2.1. Adjustable active-shunt peaking technique in 113 pre-driver designs}

114 For the radiation-tolerant purpose and as a 115 member of the data link ASIC series, the LOCld4 116 adopts the Silicon-on-Sapphire process. However, the 117 bandwidth is significantly restricted by the transit 118 frequency of this $0.25-\mu \mathrm{m}$ CMOS process. To offset 119 the bandwidth disadvantage, multi-stage 120 amplification and an adjustable active-shunt peaking

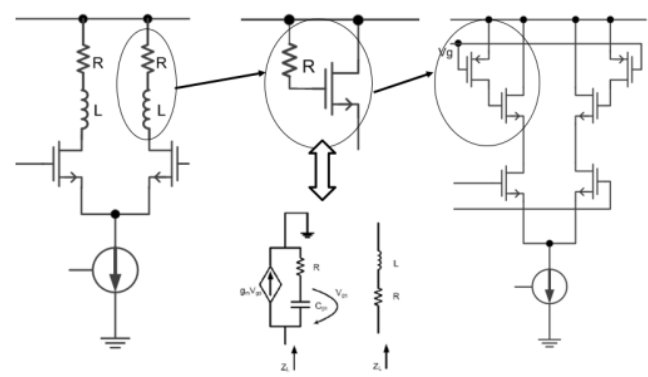

(a)

(b)

(c)

123 Fig. 3. (a) Passive inductance peaking, (b) Active shunt peaking

124 (c) Adjustable active-shunt peaking used in the LOCld4

125

126 technique based on the regular inductance peaking 127 are used in pre-drivers, as shown in Fig. 2. The pre128 drivers of each channel consist of five DC-coupled 129 stages with a tail current ratio of $1: 1.4: 2: 2.8: 4$, 130 and the total current of pre-drivers is $30 \mathrm{~mA} / \mathrm{ch}$ at 131 default settings. All four pre-drivers in different 132 channels share the same power source ( $3.3 \mathrm{~V} \mathrm{Vdd})$.

133 The inductive load added to the drain, the so134 called shunt peaking technique, is a conventional and 135 widely-used way to boost the bandwidth [12]. It 136 creates one more pole in the transmission equation of 137 the common-source amplifier to broaden the 138 bandwidth, as shown in Fig. 3(a). But the on-chip 139 passive inductance costs a large chip area. Moreover, 140 for the array driver application, the area of the single 141 channel is rigidly restricted by the VCSEL Array, 142 which has a fixed channel-to-channel interval of 250 $143 \mu \mathrm{m}$. It makes the passive inductance completely 144 unaffordable under the SOS process. The active145 shunt peaking [13], as shown in Fig. 3(b), is an 146 alternative method for the area-saving consideration. 147 The resistor R, the capacitance between source and 148 gate, and the $\mathrm{V}_{\mathrm{gs}}$-controlled $\mathrm{I}_{\mathrm{ds}}$ consist of an active 149 inductance with both equivalent conductive and 150 resistive loads. Further, the resistor R in Fig. 3(b) can 151 be substituted by a PMOSFET, which acts as a 152 voltage-controlled resistor. The final structure is 153 shown in Fig. 3(c). The PMOS gate voltage controls 154 the value of the resistance, changes the equivalent 155 value of the inductance, and makes the peaking 156 strength consequently adjustable. The programmable 157 structure enables the bandwidth optimization for each 158 chip under applications of different bit rates, and also 159 solves the instability problems of practical resistance 


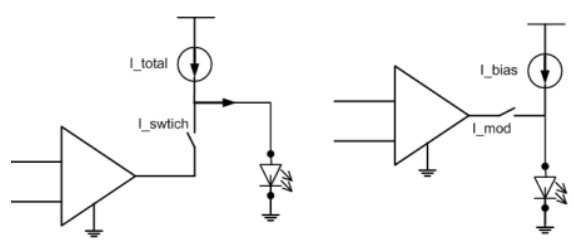

161 Fig. 4. Two regular structures of the last output driver in array 162 driver design

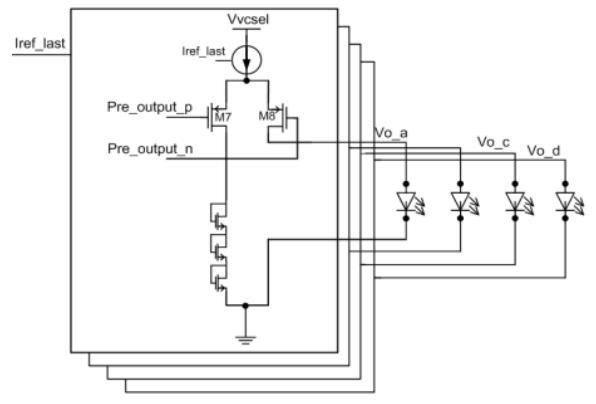

163

164 Fig. 5. Schematic of the last driver in the LOCld4

165

166 value in the process.

\section{2.2. Balanced output structure in the last driver}

168 To fit the high-density VCSEL Array, the outputs 169 of array driver need to be directly bonded to the 170 anode and cathode pads, and to deliver both the bias 171 and modulation current within the chip without any 172 peripheral circuits. Fig. 4 shows two regular designs 173 of the last output stage in an anode-driving array 174 driver. In conventional designs, the bias and 175 modulation current are always separated in a 176 subtracting or adding way with one extra current 177 source.

178 Fig. 5 shows the schematic of the last output 179 driver in the LOCld4. It removes the regular extra 180 current source, and combines the delivery of the bias 181 and modulation current in one single branch. The 182 PMOS M8 in Fig. 5 does not go deep into the cutoff 183 region. Instead, it will be partially turned on to 184 provide the bias current Ibias for the VCSEL, and 185 completely turned on to drive the VCSEL with the 186 current of $I_{\text {bias }}+I_{\text {modulation. }}$ Besides, the load of left 187 branch (internal branch) is specifically designed to be 188 balanced with the VCSEL load at right branch: three 189 diode-connected NMOS are used to simulate the 190 forward voltage and serial resistance of the VCSEL.
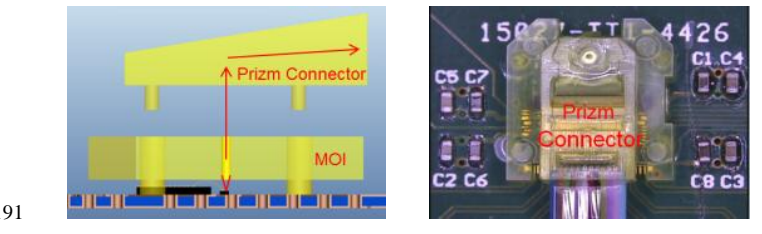

193 Fig. 6. Optical path diagram and photograph of assembled optical 194 components

195

196 The optimum value of the left-branch load can be 197 decided by a maximum common-mode rejection ratio 198 (CMRR) of the last driver stage and a minimum 199 fluctuation on the power supply (Vvcsel). The current 200 consumption of the last output driver is $13 \mathrm{~mA} / \mathrm{ch}$ at 201 default settings. The power of output driver $(3.8 \mathrm{~V}$ 202 Vvcsel) of four channels are separated within the 203 chip, and connected as one withrespective decoupling 204 capacitors outside the chip to further suppress the 205 crosstalk from the power.

\section{3. Custom array optical transmitter module: ATx}

207 Besides the array driver ASIC, the array optical 208 transmitter module is another key component for the 209 parallel optical data transmission, and also for the 210 full-channel optical evaluation of the array driver. 211 The high-density, high-precision form factor of the 212 VCSEL array compounds the difficulty in coupling 213 out parallel lights.

214 A custom array optical transmitter module (ATx) 215 [14], utilizing commercial array optical components, 216 i.e., mechanical optical interface (MOI) and Prizm 217 LightTurn Connector [7], is a versatile module that 218 can integrate different array drivers. PRIZM and 219 LightTurn are trademarks of US Conec Ltd. The 220 parallel lights of the VCSEL Array are focused by the 221 MOI, redirected by 90 degrees within the Prizm 222 Connector, and then coupled into the fiber ribbon. 223 Fig. 6 shows the optical path through the two optical 224 components. The Prizm Connector is pluggable over 225 the MOI, while the MOI needs to be epoxied onto the 226 substrate, across both the driver and VCSEL dies. 227 The focus lens of the MOI needs to be aligned to the 228 VCSEL apertures with a tolerance less than $10 \mu \mathrm{m}$. 229 This critical process is performed by a low-cost 230 reliable active-alignment method [9]. The main idea 


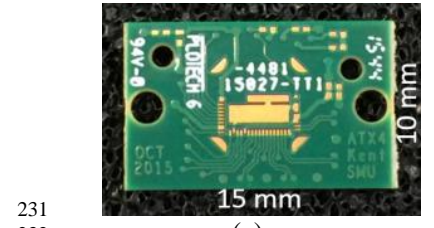

(a)

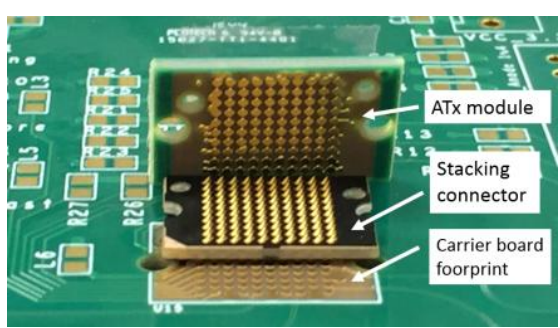

(c)

235 Fig. 7. Electrical interface of the ATx module: (a) ATx substrate 236 top, (b) ATx substrate bottom, (c) stacking structure

237

240 Fig. 8. Fully assembled ATx module on the carrier board

241

242 is to turn on the VCSEL during the alignment, and to 243 use the coupled optical power as the guide line of the 244 aligning. A three-dimensional micro-positioner and a 245 horizontal rotation stage are deployed for the 246 movement control. Under this alignment method, the 247 coupling insertion loss of each channel, the channel248 to-channel variation and the light crosstalk within the 249 adjacent channel can be stably controlled under 3 $250 \mathrm{~dB}$, under $1 \mathrm{~dB}$, and below $-50 \mathrm{dBm}$ respectively [9].

\section{3.1. Electrical interface}

252 A number of electrical interface variants have 253 been designed for different optimization purposes. 254 The first ATx prototype utilizes peripheral half-via 255 structures that can be directly soldered onto carrier 256 board. Both FR4 Print Circuited Board (PCB) and 257 Alumina ceramic substrates have been used and both 258 demonstrated sufficient transmission signal integrity

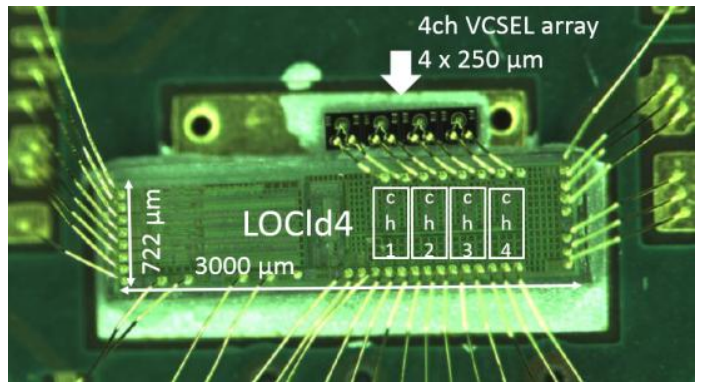

Fig. 9. LOCld4 and VCSEL array die attached and bonded

262

263 [14]. With the latest high-speed ultra-low profile 264 stacking connector [15], the electrical interface is 265 updated to a pluggable version with the footprint 266 further reduced from $19 \mathrm{~mm} \times 22 \mathrm{~mm}$ to $10 \mathrm{~mm} \times 15$ $267 \mathrm{~mm}$.

268 Fig. 7(a) (b) shows the top and bottom sides of the 269 ATx substrate. The VCSEL array die and the driver 270 die will be mounted on the top side of the substrate 271 with the MOI assembled above. The bottom of the 272 substrate adopts a footprint similar to the ball grid 273 array (BGA) package with a pitch of $0.8 \mathrm{~mm}$. The 274 stacking connector, shown in Fig. 7(c), serves as a 275 spacer between the ATx and the carrier board. Metal 276 compression contacts are arranged at both sides of 277 the connector to offer the top-to-bottom electrical 278 connection. These contacts are corresponding to both 279 the substrate footprint and the carrier board footprint. 280 Fig. 7(c) shows the overall structure of the electrical 281 interface.

282 The ATx module will be finally fixed onto the 283 carrier board together with the stacking connector 284 using two guide pins for positioning and two precise 285 screws for mechanical reinforce. Fig. 8 shows a 286 complete ATx module assembled on the carrier 287 board. The total height of the ATx module in 288 application is less than $5.3 \mathrm{~mm}$.

\section{4. Full-channel optical test of the LOCld4 after 290 integration with the ATx module}

291 With the custom array module ATx and the 292 active-alignment method, the array driver LOCld4 is 293 able to be integrated within the module and fully 294 evaluated. Fig. 9 is a photo of the LOCld4 and the 


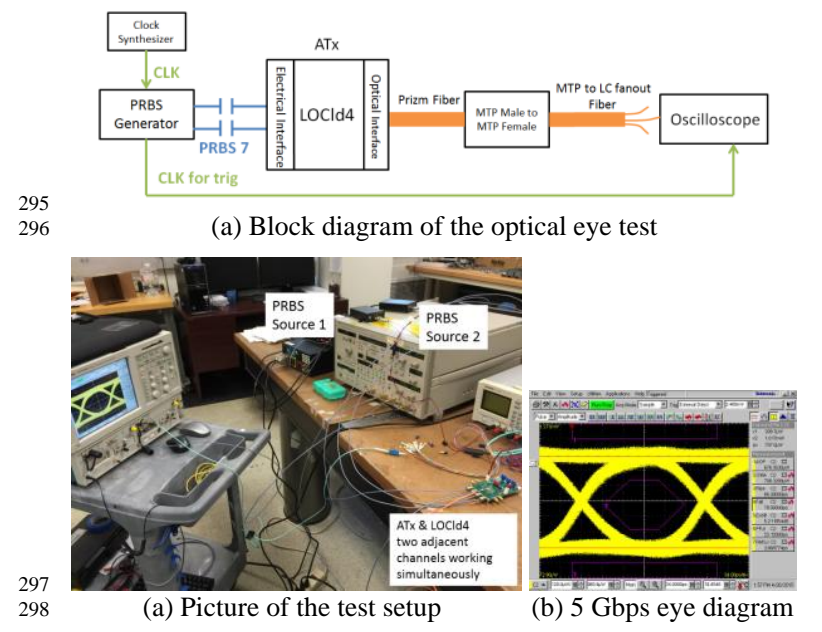

299 Fig. 10. Optical eye diagram test setup and a 5 Gbps optical eye 300

301 4-ch ULM 14G VCSEL array [16] dies attached and 302 wire-bonded on the ATx substrate.

303 Fig. 10(a) (b) shows the block diagram and the 304 setup picture of the optical eye diagram test. During 305 the test, two pseudo random binary sequence (PRBS) 306 generators were used with independent data patterns 307 and clock sources to take into account the multi308 channel crosstalk. The amplitude of the PRBS signals 309 was set to differential peak-to-peak $200 \mathrm{mV}$. At the 310 peaking strength of $2.2 \mathrm{~V}$, an optimum $5 \mathrm{Gbps}$ 311 optical eye diagram was captured as shown in the 312 Fig. 10(c), with adjacent channel working 313 simultaneously at 5 Gbps.

314 Fig. 11 shows the optimum 8 Gpbs optical eye 315 diagrams at a peaking strength of $2.4 \mathrm{~V}$. All four 316 channels were recorded with adjacent channel 317 working simultaneously at $8 \mathrm{Gbps}$, and passed the 318 eye mask test without a single violation.

319 Another BER link test was also conducted. The 320 commercial SFP+ module was used as the receiver, 321 and the LOCld4+ATx worked as the transmitter. The 322 optical output of each channel was received by the $323 \mathrm{SFP}+$ and verified with the PRBS 7 pattern. Every 324 channel was tested at $5 \mathrm{Gbps}$ and $8 \mathrm{Gbps}$ with total 325 bits up to 5E12, and not a single error was found. 326 BER less than 10E-12 was achieved at each channel 327 at the rate of $5 \mathrm{Gbps}$ and $8 \mathrm{Gbps}$ with adjacent 328 channel working at the same bit rate.

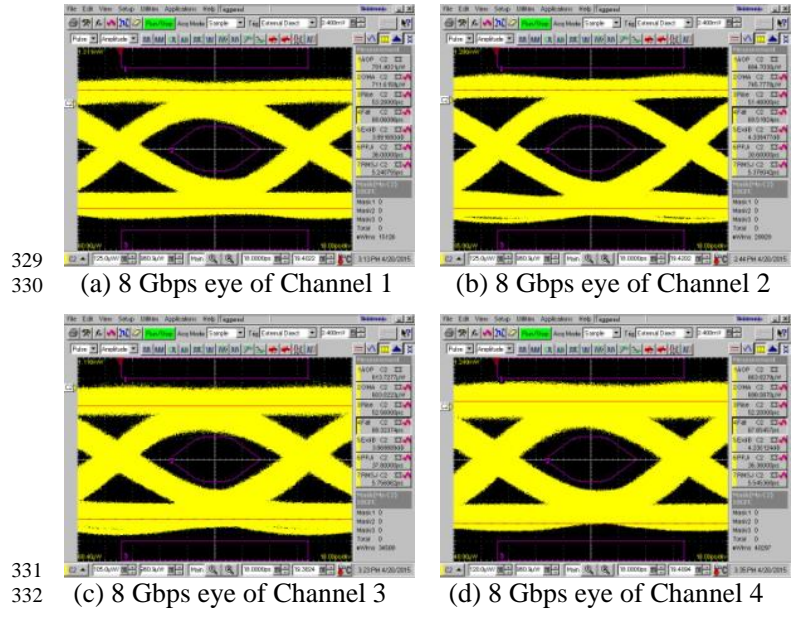

333 Fig. 11. Optical eye diagrams of all four channels at $8 \mathrm{Gbps}$

334

\section{5. Conclusion}

336 We have reported the development of a fabricated 337 VCSEL array driver ASIC and a parallel optical 338 transmitter module using commercial array optical 339 components. The array driver has been integrated 340 within the array module, and optically tested as a 341 complete parallel optical transmitter that supports $4 \mathrm{x}$ 342 8-Gbps data transmission. All four channels pass the 343 optical eye-diagram mask test at the rate of 8$344 \mathrm{Gbps} / \mathrm{ch}$ with adjacent channel working 345 simultaneously with a power consumption of 150 $346 \mathrm{~mW}$ per channel (VCSEL power included). The BER 347 less than 10E-12 transmission is achieved.

\section{Acknowledgments}

349 This work is supported by the US Department of 350 Energy Collider Detector Research and Development 351 (CDRD) data link program. The authors also would 352 like to thank Jee Libres of VLISP, Alan G Prosser of 353 Fermi Lab, Michael Wiesner of ULM and Alan 354 Ugolini of US Conec for informative discussions. 


\section{References}

356 [1] J. Chramowicz et al., Evaluation of emerging parallel optical 357 link technology for high energy physics, 2012 JINST 7 $358 \quad \mathrm{C} 01007$

359 [2] ATLAS Collaboration, ATLAS liquid argon calorimeter phase-I upgrade technical design report, CERN-LHCC-2013017, ATLAS-TDR-022, September 2013.

362 [3] ATLAS Collaboration, Letter of Intent Phase-II Upgrade, CERN-2012-022, LHCC-I-023, December, 2012.

4 [4] L. Amaral, S. Dris, A. Gerardin, T. Huffman, C. Issever, A.J. Pacheco et al., The versatile link, a common project for superLHC, Journal of Instrumentation, 2009 JISNT 4 P12003.

[5] L. Xiaoting et al., Optical Data Transmission ASICs for the High-Luminosity LHC (HL-LHC) Experiments, Journal of Instrumentation, 2014 JINST 9 C03007.

[6] F. Liang et al., The Design of 8-Gbps VCSEL Drivers for ATLAS Liquid Argon Calorimeter Upgrade, Journal of Instrumentation, 2013 JINST E02001.

3 [7] D. Childers et al., Miniature detachable photonic turn Connector for Optical Module Interface, 61st IEEE Electronic Components and Technology Conference (ECTC), 2011, pp. 1922-1927.

[8] J. Troska et al., Radiation damage studies of lasers and photodiodes for use in Multi-Gb/s optical data links, IEEE Transactions on Nuclear Science, VOL. 58, No. 6, December 2011.
381 [9] D. Guo et al., The VCSEL-based array optical transmitter (ATx) development towards 120-Gbps link for collider detector: development update, Journal of Instrumentation, 2015 JINST 10 C01034.

85 [10] T. Liu et al., Total Ionization Dose Effects and Single-Event Effects Studies of a $0.25 \mu \mathrm{m}$ Silicon-On-Sapphire CMOS technology, at 9th European Conference on Radiation and Its Effects on Components and Systems, RADECS 2007, Deauville France (2007).

90 [11] X. Li et al., 8-Gbps-per-channel radiation-tolerant VCSEL drivers for the LHC detector upgrade, Journal of Instrumentation, 2015 JINST 10 C02017.

393 [12] T. Lee, The Design of CMOS Radio-Frequency Integrated Circuits, Chap. 8, Cambridge University Press, New York U.S.A. (1998).

396 [13] E. Sackinger et al., A 3-GHz 32-db CMOS limiting amplifier for SONET OC-48 receiver, IEEE J.Solid-State Circ. 35 (2000) 1884.

399 [14] D. Guo et al., The 120Gbps VCSEL array based optical transmitter (ATx) development for the High-Luminosity LHC (HL-LHC) experiments, Journal of Instrumentation, 2014 JINST 9 C02007.

3 [15] Samtec Ltd, Ultra Low Profile Micro Array Z-Ray Catalog, F215 Rev 22JUL15.

405 [16] R. King et al., Commercial VCSELs and VCSEL arrays 406 designed for FDR (14 Gbps) optical links, Vertical-Cavity 407 Surface-Emitting Lasers XVI, Proc.SPIE, 2012 8276, 82760G. 